\title{
Respiratory epithelial cells orchestrate pulmonary innate immunity
}

\author{
Jeffrey A Whitsett ${ }^{1} \&$ Theresa Alenghat ${ }^{2}$
}

\begin{abstract}
The epithelial surfaces of the lungs are in direct contact with the environment and are subjected to dynamic physical forces as airway tubes and alveoli are stretched and compressed during ventilation. Mucociliary clearance in conducting airways, reduction of surface tension in the alveoli, and maintenance of near sterility have been accommodated by the evolution of a multi-tiered innate host-defense system. The biophysical nature of pulmonary host defenses are integrated with the ability of respiratory epithelial cells to respond to and 'instruct' the professional immune system to protect the lungs from infection and injury.
\end{abstract}

Oxidative metabolism of cells throughout the body requires the exchange of vast quantities of oxygen and carbon dioxide across the alveolar-capillary interface in the peripheral lung. Throughout life, the dynamic process of ventilation moves millions of liters of air through the highly branched conducting airways to the alveoli, the latter lined by type I and type II epithelial cells. The gracile structure of the alveoli brings epithelial cells in close apposition to pulmonary capillaries for gas exchange. While this delivers life-requiring oxygen to the systemic circulation, particles, microbes and toxicants are also brought into the respiratory tract, where they meet a multilayered physical and chemical innate host-defense system evolved to prevent their entry into lung tissue and the circulation. Innate host defenses of the conducting airway depend on its branching structure and the multiple barriers created by layers of mucus, the tight adhesions between epithelial cells and the underlying stroma, and an abundance of fluid and antimicrobial molecules that enable mucociliary clearance. Conducting airways are the conduits whose chief role is to deliver almost completely sterile, hydrated gases to the peripheral alveoli for gas exchange (Fig. 1). In sharp anatomic contrast to the airways, the alveolar region of the lungs is a unique structural environment wherein surface tension is controlled by the careful balance of fluids and unique surface active lipids and proteins that remain stable during the expansion and compression of ventilation (Fig. 2). The anatomical structures that constitute the conducting and peripheral airways serve distinct roles in the innate defense of the lungs, and the diversity of epithelial cells lining the respiratory tract contributes in unique ways to pulmonary homeostasis.

\footnotetext{
${ }^{1}$ Perinatal Institute, Division of Neonatology, Division of Perinatal Biology and Division of Pulmonary Biology, Cincinnati Children's Hospital Medical Center and the University of Cincinnati College of Medicine, Cincinnati, Ohio, USA. ${ }^{2}$ Division of Immunobiology, Cincinnati Children's Hospital Medical Center and the University of Cincinnati College of Medicine, Cincinnati, Ohio, USA. Correspondence should be addressed to J.A.W. (jeff.whitsett@cchmc.org).
}

Received 8 September; accepted 30 October; published online 18 December 2014; doi:10.1038/ni.3045.

\section{Secreted products of lung epithelial cells}

The conducting airways of the lungs, from the trachea to terminal bronchioles, are formed by budding and branching of endodermderived tubules by the process of branching morphogenesis ${ }^{1}$. In human lungs, cartilaginous airways extend deep into the lung parenchyma and are surrounded by an abundance of submucosal glands that secrete fluids, mucins and other host-defense proteins into the airways. The human trachea, bronchi and bronchioles are lined mainly by a pseudostratified epithelium whose surface is dominated by ciliated cells (Fig. 1a,b). The highly ciliated nature of primate airways is distinct from that in the mouse and other rodents, in which secretory cells are much more abundant. Basal cells located beneath the surface epithelium serve as progenitors of both ciliated cells and secretory cells and have a critical role in regeneration of the airway epithelium following injury. A diversity of other epithelial cell types, including those in submucosal glands and other nonciliated respiratory cells, serve as progenitors following lung injury ${ }^{2,3}$. Although ciliated cells are the predominant surface cells, secretory cells, including serous, club, neuroendocrine and goblet cells, are found in relatively low numbers in normal airways. The diverse cell types lining the lung synthesize and secrete an abundance of fluids, antimicrobial proteins and mucins, and their numbers and secretory activity are influenced by injury and infection. Submucosal glands are also lined by many cell types, including myoepithelial, serous, goblet, basal and ciliated cells, that together secrete fluids and other host-defense proteins onto the airway surface, at baseline and in response to environmental stimuli (Fig. 1). Conducting airways and submucosal glands secrete an array of hostdefense molecules involved in the aggregation, trapping and killing of microbes. To name a few, human $\beta$-defensins, lysozyme, lactroferrin, cathelicidin LL37 and surfactant proteins A and D are expressed by airway epithelial cells and are regulated by exposure to pathogens, toxicants and cytokines ${ }^{4}$.

In sharp contrast to the diversity of cell types that produce innate defense proteins in conducting airways, only two cell types line the alveoli. Squamous type I alveolar cells cover approximately $90 \%$ of the alveolar surface in the adult lungs and interact closely with endothelial cells of pulmonary capillaries (Fig. 2a,b). The other cells that line the 

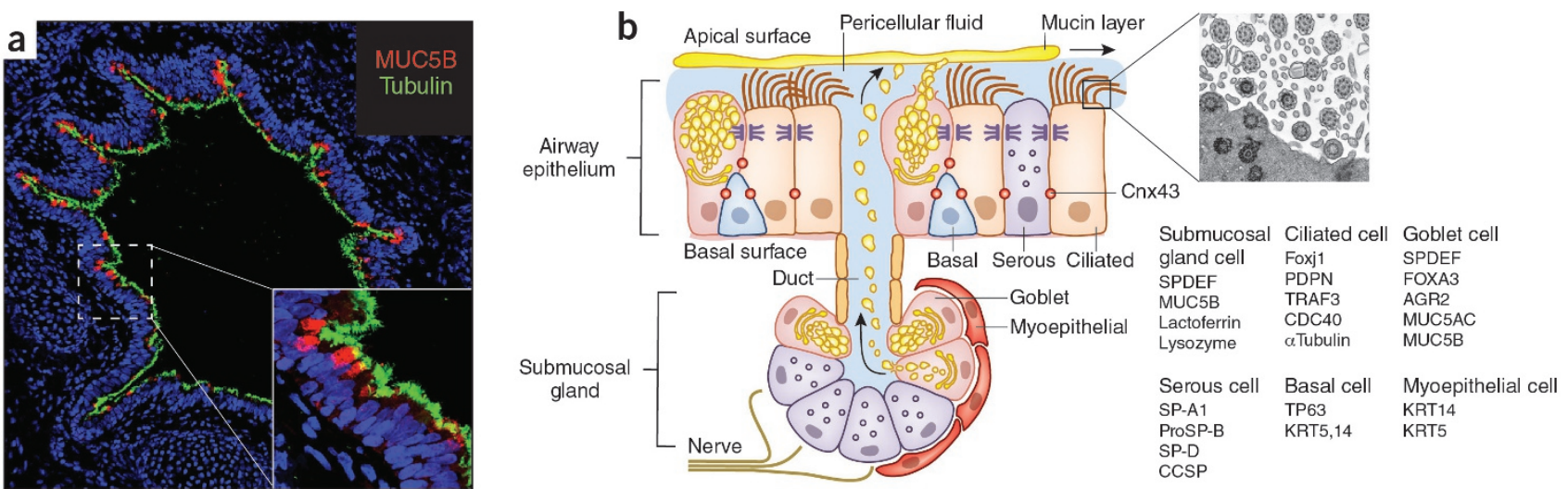

Figure 1 Structure and function of the innate host defenses in conducting airways. Cartilaginous airways from the terminal bronchioles to the trachea are lined by a pseudostratified epithelium, whose surface is lined by ciliated and secretory cells, that together with submucosal glands, secrete mucins and other host-defense proteins into the periciliary fluids $(\mathbf{a}, \mathbf{b})$. Various transcription factors and associated proteins (b, bottom right) are selectively expressed in distinct subsets of epithelial cells lining the airways and submucosal glands. Secreted mucins (blue), such as MUC5AC and MUC5B, produced by goblet cells create a hydrated mucus gel $(\mathbf{c}, \mathbf{d})$ that binds particles and pathogens that are moved by the periciliary brush (b) up the airway for clearance from the lungs. Epithelial cells lining the airways and submucosal glands (b,d) create tight epithelial barriers and secrete a diversity of host-defense proteins that recognize microbial pathogens, which enhances the uptake and killing of

C

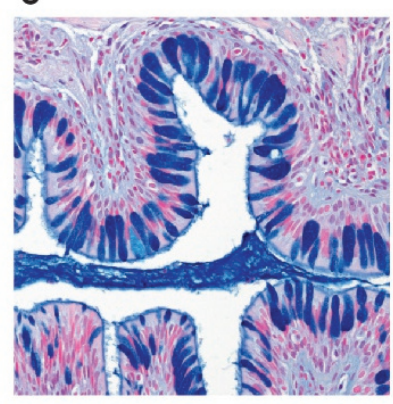

d

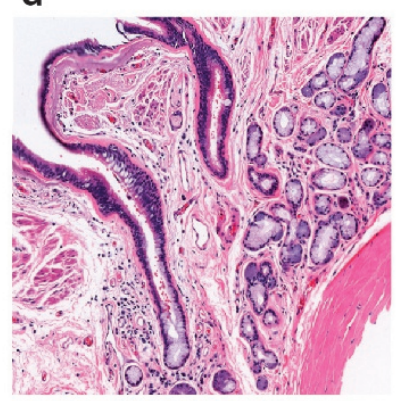
those pathogens by professional cells of the immune system. The biophysical scaffolds created by the mucus gel, tight cell-cell junctions and communication among respiratory epithelial cells provide multiple barriers to infection. The secretion of fluid and mucus is coordinated with the directional beating of cilia (ultrastructure in electronmicrograph in $\mathbf{b}$ ) mediated by $\mathrm{Cnx} 43$.

alveoli are cuboidal type II epithelial cells. These are readily recognized by their abundance of lipid-rich lamellar bodies, microvilli on their apical surfaces and their expression of proteins that mediate surfactant homeostasis, such as ABCA3, SP-A, SP-B, SP-C and SP-D ${ }^{5}$. Type II alveolar cells have critical roles in the synthesis of surfactant lipids and proteins required for the reduction of surface tension to prevent collapse of the lungs (atelectasis) during the ventilatory cycle and to serve as the main progenitor cells during repair of the alveoli, functioning as self-renewing cells and precursors of type I cells ${ }^{6}$.

\section{Barrier functions of the respiratory epithelium}

Respiratory epithelial cells create multiple barriers mediated by their secretory products, surface glycocalyces and membranes, and intercellular junctional proteins, the last mediated by claudins, connexins, paranexins, adhesions and zonula occludins that are linked to the actin cytoskeleton and provide structural integrity to the respiratory epithelium. Apical junctional complexes are formed by tight and adherens junctions created in part by homotypic and heterotypic binding among the many claudins expressed in pulmonary cells. Claudin 3 (Cld3), Cld4 and Cld18, which all have high expression in alveolar epithelial cells, create interlocking structures that form tight junctions to link alveolar epithelial cells. The importance of Cld18 in innate host defense is supported by findings demonstrating loss of barrier function and susceptibility of $C l d n 18^{-1-}$ mice to infection and injury by pathogens and proteases ${ }^{7}$. Loss of Cld3 and Cld4 in alveolar epithelial cells increases alveolar-capillary permeability and inhibits fluid clearance ${ }^{8}$. Disruption of tight junctional complexes increases epithelial permeability and inflammation in both conducting airways and alveoli, which contributes to the pathogenesis of asthma, acute respiratory distress syndrome and barotrauma-induced lung injury ${ }^{7,9-13}$.

\section{Mucus and mucociliary clearance}

The luminal surfaces of the conducting airways, like those of the gastrointestinal and reproductive organs, are in direct contact with microbes and particles that must be removed or accommodated, to prevent their access to underlying epithelial cells. Cell-associated and secreted mucins serve to create a barrier, and biophysical 'rafts' of polymeric glycoconjugates bind to and transport pathogens from the conducting airways. Mucins are large glycoproteins that share an abundance of repeated threonine-rich domains decorated by a rich array of complex O-linked polysaccharides. Mucins that are 'tethered' to epithelial cells (for example, MUC4, MUC13, MUC16 and MUC21) create a direct host defense barrier at the epithelial surface that can be shed by pathogen or host-associated proteases, which releases offending microbes to the mucociliary 'escalator' for removal. The secreted airway mucins (MUC5B, MUC5AC and MUC2), which are encoded by genes located in a contiguous region of human chromosome 11, form a mucous gel that disrupts bacterial aggregation and binds microbial pathogens and prevents them from adhering to cell surfaces, which enhances their clearance by the mucociliary 'escalator' 14,15 . Although secreted airway mucins are notably associated with goblet cells that contain abundant mucin granules, mucins are also produced by club cells (MUC5ac) and alveolar cells (MUC1) in the conducting and peripheral airways and by goblet cells in submucosal glands; the last site expresses an abundance of MUC5B. Mucins undergo extensive post-translational folding and processing via both their carboxy-terminal domains and their aminoterminal domains, which generates linear and multimeric networks that create a gel-like lattice of polymeric proteins that is moved up the airway by the beating of cilia ${ }^{16}$. The abundance and types of mucus secreted by airway epithelial cells vary during development and along the proximalperipheral airway of the lungs and are highly responsive to particles 


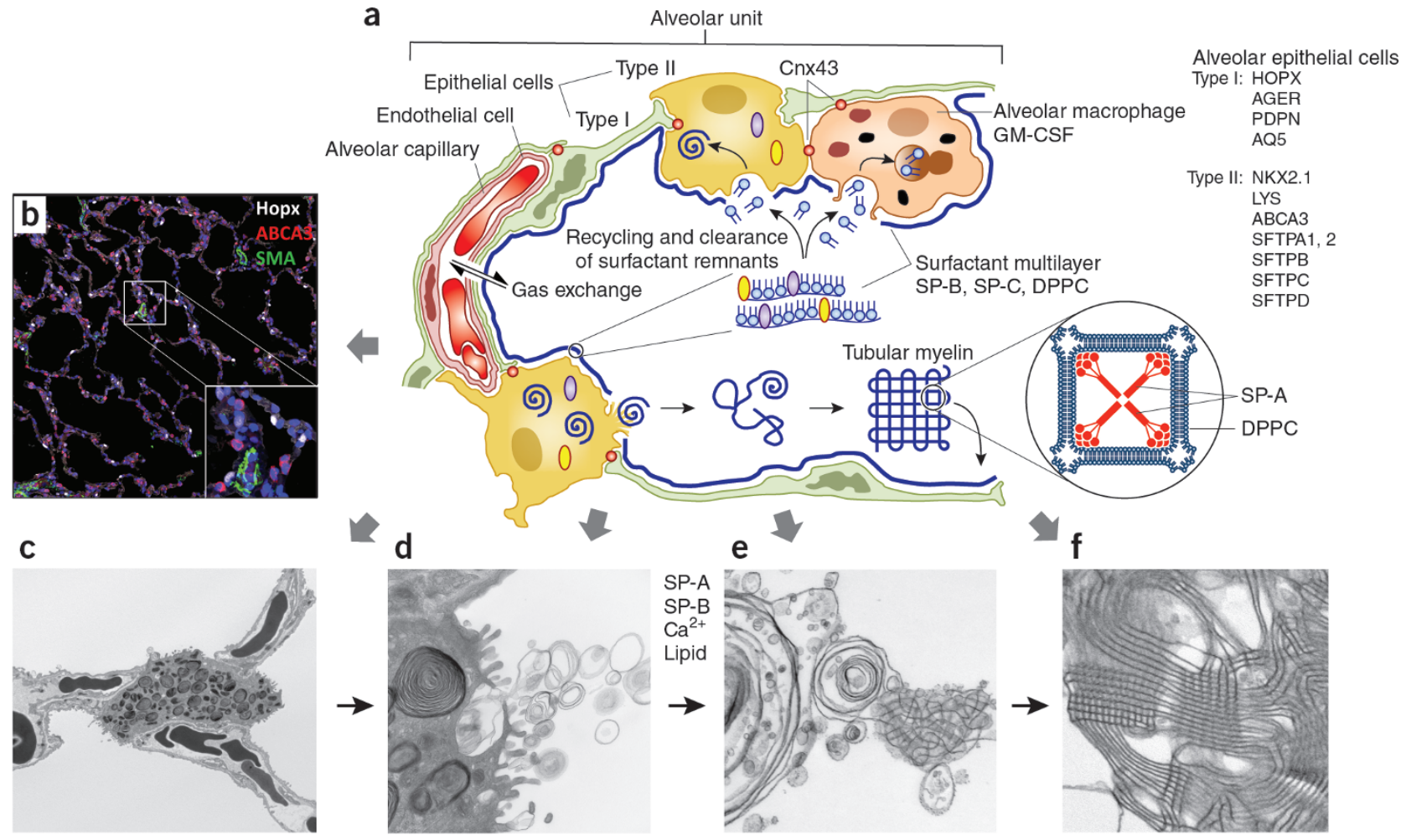

Figure 2 Integration of surfactant function and innate host defenses in the alveoli. Gas exchange is mediated by the close apposition of type I and type II epithelial cells to the endothelial cells of pulmonary capillaries, which creates an extensive surface area whereon environmental gases create collapsing forces at the hydrated surfaces of the alveoli $(\mathbf{a}, \mathbf{b})$. Hopx is a transcription factor selectively expressed in type I cells, and ABCA3 is a surfactant lipid transporter specific for type II epithelial cells in the alveoli (b). Antibody to smooth muscle actin ( $\alpha$-SMA) stains bronchiolar and vascular smooth muscle. Surface tension is diminished by pulmonary surfactant lipids and proteins secreted by type II epithelial cells (c-e) that remain stable during the dynamic compression and expansion of the lungs during ventilation. The biophysical activities of surfactant are integrated with alveolar host-defense functions that are mediated by the structural components of surfactant that have intrinsic antimicrobial activity. Tubular myelin (a,f), formed by surfactant proteins SP-A and SP-B, and lipid create a highly structured reservoir of surfactant and host-defense proteins that interact with alveolar macrophages and other cells of the immune system to bind to and remove microbial pathogens and 'instruct' inflammatory cells to mount appropriate host-defense responses (b). Alveolar epithelial cell and alveolar macrophages directly interact via $\mathrm{Cnx} 43$ channels to modify local inflammatory signals and regulate the expression of cytokines and chemokines in response to pathogens. The sizes of surfactant pools are maintained by the synthesis, secretion and reuptake of lipids and proteins by alveolar epithelial cells and by the catabolic activities of alveolar macrophages via processes regulated by GM-CSF that together maintain near sterility of the alveoli (a).

and pathogens ${ }^{15,17}$. While increased synthesis and secretion of airway mucus is often a noisome accompaniment to the resolution of infection or environmental exposure, mucins serve to maintain airway homeostasis and the removal of pathogens and cellular debris during recovery from infection or injury. Excessive goblet cell differentiation and mucus hyperproduction are characteristic of chronic airway diseases, such as cystic fibrosis, chronic obstructive pulmonary disease (COPD), bronchiectasis (distension of the bronchi) and asthma (Fig. 1c). The transcriptional machinery that regulates goblet cell metaplasia and mucus production is intrinsically linked to inflammatory signaling via the Toll-like receptors (TLRs) and transcription factors of the IRF and NF- $\kappa \mathrm{B}$ families and cytokine signaling via the Jak kinase-STAT transcription factor pathway in respiratory epithelial cells ${ }^{15,18-20}$ (Fig. 3).

\section{MUC5AC and MUC5B in pulmonary innate immunity}

MUC5B and MUC5AC are the most abundant secreted mucins in conducting airways. While they serve critical roles in the formation of the hydrated mucus gel and the tethering other host defense proteins, mucins selectively bind to and disrupt the aggregation of microbial pathogens, which prevents the pathogens from entering the periciliary layer and blocks their access to underlying epithelial cell surfaces.
Insights into the distinct roles of MUC5AC and MUC5B in the homeostasis of airway epithelial cells have been provided by the study of transgenic mice that lack or express MUC5AC and MUC5B. Mice deficient in MUC5AC, which is normally expressed both in the intestine and in the lungs, survive but do not clear the helminth Trichiuris muri after infection, a result probably related to impaired host defense in the intestine ${ }^{21}$. Surprisingly, $\mathrm{Muc5ac} \mathrm{c}^{-/-}$mice fail to recruit neutrophils following acute lung injury and are less susceptible to ventilator-associated inflammation $^{22}$. Increased expression of MUC5AC in airway epithelial cells confers resistance to bacterial infection but does not cause mucus metaplasia or airway obstruction ${ }^{23}$. In sharp contrast to the relatively mild altered pulmonary phenotypes associated with lack of MUC5AC, Muc5 $b^{-1-}$ mice develop severe pulmonary pathology and chronic bacterial infection with obstruction to airflow and inflammation ${ }^{24} . \mathrm{Muc}^{\mathrm{S}} \mathrm{b}^{-/-}$mice fail to clear particles and pathogens from the airway, which indicates the critical role of MUC5B in lung homeostasis. Lack of MUC5B inhibits inflammatory responses, suppressing interleukin 23 (IL-23) and causing the accumulation of atypical, apoptotic alveolar macrophages whose phagocytosis and clearance of Staphylococcus aureus are impaired. The critical role of the mucus layer in lung innate defense is highlighted by the severe lung disease caused by mutations in the human gene encoding 
Figure 3 Signaling via PAMPs and DAMPs in respiratory epithelial cells and downstream host-defense responses. PAMPs derived from commensal microbes or respiratory pathogens and DAMPs generated from cell stress and/or death within both the conducting airways and alveoli are recognized via membraneassociated or cytosolic PRRs expressed in respiratory epithelial cells. The binding of ligands to these receptors results in the activation of epithelial cell-intrinsic signaling pathways (via MAPK, IRFs, reactive oxygen species (ROS) and NF-KB) and subsequent production of cytokines, chemokines and antimicrobial proteins that recruit and activate cells of the innate and adaptive immune systems and regulate barrier function. These same recognition pathways in epithelial cells can stimulate autophagy, phagocytosis and the clearance of necrotic cells and pathogens and thus further influence local inflammatory responses. dsRNA, double-stranded RNA.

cystic fibrosis transmembrane conductance regulator $(C F T R)$, which cause lung disease in people with cystic fibrosis. Mutations in CFTR inhibit cAMP-induced transport of $\mathrm{Cl}^{-}$and $\mathrm{HCO}_{3}{ }^{-}$by epithelial cells that line the airways and submucosal glands, which causes mucus thickening (inspissated mucus), failure of mucociliary clearance and chronic bacterial infection. The lack of detachment and spreading of newly secreted mucus from submucosal glands in pigs in which the gene encoding CFTR is targeted supports a model in which epithelial cells lining submucosal glands do not adequately hydrate the mucus gel, impairing mucociliary clearance ${ }^{25,26}$. Colonization of the thick, dehydrated mucus in the diseased airways with Pseudomonas aeruginosa creates biofilms that serve as reservoirs for chronic, polymicrobial bacterial infection ${ }^{27}$. P. aeruginosa flagellin induce airway fluid secretion and mucociliary clearance via a process dependent on CFTR, which provides a plausible mechanism by which patients with cystic fibrosis are susceptible to infection ${ }^{28}$.

\section{Goblet cell differentiation is linked to innate signaling}

Goblet cells and other secretory epithelial cells that line conducting airways are derived from epithelial progenitors, including basal cells, club cells and other airway cells whose differentiation is determined by the transcription factor SOX2 during morphogenesis of the embryonic conducting airways ${ }^{2}$. The fate of epithelial cells is influenced by signaling via receptors of the Notch family that establishes the complexity of cellular niches and creates the stereotyped 'cobblestone' patterning of airway epithelial surfaces ${ }^{29-31}$. The sites and extent of airway goblet cell differentiation vary during development and are strongly influenced by environmental exposures, infection and inflammation. The differentiation of airway goblet cells from basal cells and other airway cells requires SPDEF, a transcription factor that regulates genes encoding a network of molecules involved in mucus biosynthesis and secretion, including MUC5AC, MUC5B, MUC16, Foxa3 and enzymes and proteins that regulate the folding, packaging and processing of mucins ${ }^{32}$. The expression of secreted mucins is induced by the transcription factors STAT6, CREB, SP-1 and AP-1, the NAPDH oxidase component NOX-4, mitogen-activated protein (MAP) kinases and the transmembrane protein TM16A ${ }^{32-34}$; on the other hand, it is inhibited by the transcription factors Foxa2 and TTF-1 (ref. 35). Studies also indicate an important role for the calcium channel CLCA1 and MAPK13 in IL-13-induced mucus production ${ }^{36}$.

\section{SPDEF and Foxa3 'instruct' immunological signaling}

Although transient mucus hyperproduction and goblet cell metaplasia are normal responses to acute lung infection or injury, goblet cell differentiation and mucus hypersecretion are associated with increased susceptibility to infection and airway obstruction. In airway epithelial cells, SPDEF and Foxa3 are induced by infection with rhinovirus and exposure to interferons and type 2-related cytokines ${ }^{37}$. SPDEF and Foxa3 enhance $T$ helper type $2\left(\mathrm{~T}_{\mathrm{H}} 2\right)$ immune responses, inducing the cytokines TSLP, IL-33 and IL-17 and inhibiting interferon responses to rhinovirus. SPDEF also binds to the signaling adapters TRIF and MyD88, inhibiting NF-кB- and interferon-mediated pathways in airway cells $^{38}$. Whereas this transcriptional network enhances the mucus production and mucociliary clearance needed to restore airway homeostasis following acute injury to the normal lungs, chronic expression of SPDEF and Foxa3 and the associated mucus hyperproduction can contribute to pulmonary dysfunction and recurrent infections in patients with COPD, asthma and/or cystic fibrosis.

\section{Role of motile cilia in innate defense of the airways}

The transport of particles and pathogens from the lungs depends on the coordinated directional movement of the mucus gel driven by the actions of the abundant ciliated cells that line human and primate lungs (Fig. 1). Ciliated cells represent the vast majority of luminal epithelial cells in cartilaginous airways in human lungs. Motile cilia are formed by the assembly of microtubules associated with dynein arms to form axonemes of motile cilia that are powered by ATP 39,40 . Primary and secondary factors that cause ciliary dysfunction impair mucociliary clearance, which leads to recurrent infections associated with common pulmonary diseases. The importance of ciliary function is emphasized by the severely detrimental clinical phenotypes associated with inherited disorders of motile cilia, collectively called 'primary ciliary 
dyskinesia' $(\mathrm{PCD})^{39,40}$. PCD is a relatively rare disorder with an estimated prevalence of 1:15,000; it is caused by a growing number of mutations, including mutations of more than 25 distinct genes, usually inherited as autosomal recessive Mendelian disorders. Most genes with mutations that cause PCD encode proteins associated with outer and inner dynein arms, although mutations in genes encoding molecules that regulate the cytoplasmic assembly of cilia have been identified. Patients with PCD commonly present as newborns with transient respiratory distress or, later, with chronic sinusitis-otitis, cough, bronchiectasis and/or infertility, variably associated with abnormal right-to-left organ patterning, situs inversus or situs ambiguous (mirrored or abnormal distribution of visceral organs) $)^{39,40}$. Decreased production of nitric oxide by the airway epithelial cells, ultrastructural abnormalities in cilia, chronic multibacterial infection, bronchiectasis and the identification of biallelic mutations in genes encoding molecules critical for the formation and function of cilia distinguish the severe lung disorder of PCD from that of cystic fibrosis ${ }^{40}$. Bacterial cultures of sputum from patients with PCD often contain S. aureus, Streptococcus pneumoniae, Haemophilus influenzae and non-tubercular myocobacteria, and chronic infection with $P$. aeruginosa occurs at older ages in patients with PCD than in patients with cystic fibrosis ${ }^{40}$. Increased production of IL-1, IL-6 and tumor-necrosis factor has been observed during pulmonary infection with $S$. pneumoniae in mouse models of PCD caused by loss of the genes encoding the PCD-related proteins Pcd 1 and Spdef2, which links ciliary dyskinesia to innate defense responses ${ }^{41}$. Secondary dysfunction of the cilia is associated with abnormalities in airway hydration and mucus hyperproduction associated with cystic fibrosis, COPD and cigarette smoking that impair ciliary function and mucociliary clearance, which leads to recurrent infections, bronchiectasis and airway obstruction.

The biogenesis of motile cilia is directly linked to the differentiation of ciliated cells from basal cells and other airway progenitors and is driven by highly conserved transcriptional programs that are dependent on members of the regulatory factor X family, multicillin and Foxj1 (ref. 42). The number of ciliated and non-ciliated cells varies among species and along the proximal-peripheral axis of conducting airways and is strongly influenced by toxicants and inflammatory processes, such as cigarette smoking. Ciliary length and dysfunction are linked to autophagy and are probably involved in the pathogenesis of airway disease in COPD ${ }^{43}$.

\section{Connexins coordinate respiratory epithelial cells}

Precise coordination of ciliary beat frequency and directionality along the airway epithelium is needed to move mucus above the periciliary fluid layer and toward the larynx. Ciliary beat frequency responds to mechanical stress and neurochemical and inflammatory signals that induce intracellular calcium transients coupled by the intercellular movement of inositol trisphosphate. Paracrine signals, including those by purinergic and other signaling receptors on the basolateral and apical surfaces of respiratory epithelial cells, influence ciliary function. Stimulus-induced responses are rapidly exchanged among ciliated cells via gap junctions. While a diversity of gap junctions proteins is involved in coupling epithelial cells and non-epithelial cells in the lung, the connexin Cnx43 has a critical role in intracellular communications among cells lining conducting airways in regulating the activity of cilia that are closely integrated with inflammatory signaling ${ }^{44}$. Cnx 43 mediates calcium-dependent signaling following the activation of TLR2 by $P$. aeruginosa to activate NF- $\mathrm{KB}$ and the epithelial secretion of cytokines (CXCL8), which recruits neutrophils to sites of pulmonary infection ${ }^{45}$.

\section{Role of the surfactant system in innate host defense}

Inherent in their structure and function, the alveoli create an extensive surface where type I cells and capillary endothelial cells come into close apposition to mediate the efficient exchange of oxygen and carbon dioxide (Fig. 2). The elastic structure of the alveoli, as well as the necessity to dynamically diminish surface tension at the gas-liquid interface at the alveolar surface, presents unique host-defense and biophysical challenges, the failure of which leads to infection, tissue damage, alveolar capillary leak and potential catastrophic collapse of the lungs (atelectasis) that impairs ventilation, processes that underlie the pathogenesis of acute respiratory distress syndromes in infants and adults. The repertoire of host-defense proteins and processes in surfactant are uniquely suited for alveolar function and are distinct from those in conducting airways. Innate host defenses in the alveoli are constrained by the challenges of producing proteins whose structures and functions do not impair but instead enhance the surface activities of lipids (Fig. 2). Surfactant proteins contribute to the structure, regulation, and function of pulmonary surfactant and have intrinsic host-defense properties $^{5}$. Pulmonary surfactant is composed mainly of lipids, as well as surfactant proteins at lower concentrations, that together serve critical roles diminishing surface tension in the alveolus during the dynamic changes in lung volumes generated during the ventilatory cycle ${ }^{5}$. The surfactant proteins SP-A and SP-D are well-conserved members of the collectin family of innate host-defense proteins and have high expression by type II alveolar epithelial cells. The carboxy-terminal lectin-like domains of SP-A and SP-D bind with varying specificity to a diversity of pathogen-associated molecular patterns (PAMPs), including complex carbohydrate surfaces of common respiratory viruses, bacterial and fungal pathogens, and associated toxins, which enhances the opsonization and killing of the pathogens by alveolar macrophages and regulates the activities of macrophages, neutrophils and lymphocytes ${ }^{46,47}$. The surfactant proteins SP-B and SP-C are synthesized and proteolytically processed by type II alveolar epithelial cells, which create the small hydrophobic peptides needed for the spreading and stability of surfactant lipids.

\section{Tubular myelin creates a host-defense scaffold}

Tubular myelin is a highly structured lipid-protein complex with distinct roles in alveolar homeostasis; it provides an extracellular reservoir of surfactant lipids that move to the multilayered films at the air-liquid interface to reduce surface tension and a scaffold made of and hosting innate defense proteins (Fig. 2). Surfactant lipids show enrichment for dipalmitoylphosphatidylcholine, and other lipids, such as phosphatidylglycerol, have intrinsic antimicrobial activities ${ }^{48,49}$. The formation of tubular myelin requires SP-A and SP-B produced by type II epithelial cells (Fig. 2). The characteristic ultrastructure of the tubular myelin is imparted by binding of the carboxy-terminal domain of SP-A in the corners of the lipid lattice and the oligomerization of its collagenous domains across its diagonal. Tubular myelin hosts other innate defense proteins, including lysozyme and SP-C, that have distinct antiviral and antimicrobial activities in addition to their ability to enhance surfactant activity. SP-B, a cationic protein, is required for surfactant function and enhances the killing of bacteria at acidic $\mathrm{pH}$ within alveolar macrophages ${ }^{50,51}$. SP-C, a small proteolipid-like peptide required for normal surfactant activity, also binds endotoxin, and this seems to have a protective effect, because SP-C-deficient mice are susceptible to pulmonary inflammation following exposure to endotoxin ${ }^{52,53}$. Similarly, SP-C-deficient $\left(S f t \mathrm{c}^{-/-}\right)$mice are susceptible to lung injury during pulmonary infection with respiratory syncytial virus ${ }^{54}$. In addition, mice deficient in SP-A or SP-D are highly susceptible to pathogenic bacteria and viruses and their products ${ }^{55}$. Surfactant-associated innate defense molecules bind, aggregate and/or directly kill microbial pathogens and enhance their clearance by professional cells of the immune system and minimize inflammation. 


\section{Alveolar macrophages in lung immunological homeostasis}

Alveolar concentrations of surfactant lipids and proteins are controlled by de novo synthesis, reuptake and recycling by alveolar type II cells and by the catabolic activity of alveolar macrophages (Fig. 2a). Studies of mice with deletion of $C s f 2$, which encodes the macrophage growth and differentiation factor GM-CSF, have provided critical insights needed to link surfactant catabolism and the innate immune defense functions of alveolar macrophages ${ }^{56}$. The recruitment and differentiation of the precursors of alveolar macrophages depend on GM-CSF produced by pulmonary parenchymal cells that activates signaling via the receptor for GM-CSF to regulate PU.1, an Ets transcription factor that activates genes encoding molecules required for alveolar macrophage differentiation, surfactant protein and lipid catabolism, and appropriate innate defense responses ${ }^{57,58}$. Antibodies to GM-CSF impair the clearance of surfactant by macrophages, which results in acquired pulmonary alveolar proteinosis $(\mathrm{PAP})^{56}$. Acquired PAP is corrected in mice and human patients by inhalation of GM-CSF ${ }^{59}$. Biallelic mutations in CSF2RA and CSF2RB, which encoding receptors for GM-CSF, have been identified in patients with hereditary PAP ${ }^{60-62}$. The provision of progenitors of alveolar macrophages into which the normal receptors of GM-CSF have been transduced restores both the size of the pulmonary surfactant pool and macrophage function in $\mathrm{Cs} f 2 \mathrm{ra}^{-1-}$ mice ${ }^{63}$. Impairment of GM-CSF signaling is associated with susceptibility to bacterial and viral infections in mouse models and with atypical clinical infections in people with PAP ${ }^{64}$. Resident macrophages and dendritic cells serve pleotropic roles in the alveoli by responding to pathogens, sensing antigens and activating innate and acquired immunity. Alveolar macrophages clear apoptotic cells during infection and inhibit inflammation. Remarkably, Cnx43 mediates direct physical interactions among type II and type I cells and alveolar macrophages, coupling cell signaling that modulates local inflammatory responses to injury ${ }^{9,65}$.

\section{Apoptosis, the unfolded protein response and autophagy}

Although airway epithelial cells are highly responsive to external stimuli, the ongoing synthetic and metabolic requirements for innate host defenses place these cells in a precarious balance between expenditure of energy and the cell activities needed to maintain homeostasis and those needed to respond to infection and injury. Multiple pathways that regulate apoptosis, reactive oxygen species, DNA damage, autophagy and the unfolded protein response (UPR) serve to integrate cell survival with the need to respond to and clear pathogens and infected cells ${ }^{66-68}$. The clearance of infected cells is mediated by activities of the acquired immune system, such as $\mathrm{CD} 8^{+} \mathrm{T}$ cells, and by epithelial cell-autonomous mechanisms; however, studies have demonstrated that subsets of epithelial cells in infected conducting airways can survive clearance by the immune system, and their survival contributes to ongoing inflammation following infection with influenza A virus ${ }^{69}$. The UPR and other stress responses link the folding of proteins to inflammatory signaling and cell survival that is well adapted to the recognition of non-host cell proteins encoded by viruses and other pathogens. The synthesis of misfolded proteins encoded by microbial pathogens creates cell stress and inflammation. The UPR activates apoptosis and necrosis to remove infected epithelial cells and pathogens. Although it is well established that alveolar macrophages ingest infected and apoptotic cells by phagocytosis, studies have also demonstrated that similar phagocytic activity by respiratory epithelial cells results in inhibition of inflammation ${ }^{70}$. The battle between viruses and the synthesis of non-mammalian proteins and the inherent metabolic and synthetic requirements of the respiratory epithelial cells have driven adaptation by both pathogens and hosts during evolution. The UPR integrates the metabolic challenge of producing large amounts of complex proteins of the innate immune sys- tem required for host defense with the ability to recognize and respond to proteins encoded by invading pathogens to signal inflammation or cell death. Spontaneous activation of the UPR and apoptosis of airway epithelial cells occurs in patients with familial interstitial lung disease (ILD) and sporadic idiopathic pulmonary fibrosis ${ }^{71-73}$. In type II epithelial cells, mutated versions of genes encoding the surfactant proteins SFTPA and SFTPC and the surfactant lipid transporter ABCA3 result in induction of the UPR and epithelial cell injury involved in the pathogenesis of familial ILD in children and adults ${ }^{71,72,74}$. Genome-wide association studies have also linked alleles on the chromosome 11 locus that encodes the gel-forming mucin MUC5B with susceptibility to idiopathic pulmonary fibrosis ${ }^{75}$. Allergens, $\mathrm{T}_{\mathrm{H}} 2$ cytokines and exposure to virus enhance both mucus production and endoplasmic reticulum stress, as indicated by the induction of IRE-1 $\beta$, a molecule required for mucin biosynthesis ${ }^{76}$. Ongoing epithelial injury related to misfolded proteins causes pulmonary inflammation, remodeling and loss of lung function. Likewise, impairment of surface activity, such as that caused by selective loss of the production of SP-B or SP-C by type II epithelial cells, is sufficient to impair both alveolar barrier and macrophage function and cause inflammation and tissue injury ${ }^{77,78}$.

\section{Responses to commensal and pathological microbes}

The respiratory epithelium is constantly exposed to environmental microorganisms and their byproducts. Although long considered sterile, both the upper and lower regions of the normal lung in fact harbor a relatively low abundance of 'commensal' bacteria whose diversity exhibits general similarity to that of taxonomic species found in the oropharyn $\mathrm{x}^{79,80}$. The clearance of microbial pathogens and adaptation to a commensal flora are mediated by the mucociliary barrier and carefully gated innate and acquired immune responses that minimize inflammation and maintain tissue homeostasis. Exposure of the respiratory tract to the commensal microbiota and to viral infection early in life strongly influences adaptive and innate immune responses to subsequent exposures, regulates the acquisition of $\mathrm{T}_{\mathrm{H}} 1$ and $\mathrm{T}_{\mathrm{H}} 2$ immunity during development, and affects asthmatic and non-asthmatic responses later in life ${ }^{81,82}$. Respiratory epithelial cells have an essential important role in the initial recognition and amplitude of the inflammatory responses to microbial pathogens through activation of TLRs and receptors of the NLR family, signaling via Jak-STAT, NF-KB and IRFs, and subsequent production of cytokines and chemokines. How specific and stochastic signals from commensal and microbial pathogens are integrated for appropriate responses to stimuli while minimizing tissue injury and directing appropriate immune responses remains an intriguing question in mucosal immunity. The lungs of smokers and patients with cystic fibrosis, COPD and/or asthma display disruption of normal mucociliary clearance, which might alter the quantity and diversity of bacterial populations in the lung, and underlie clinical exacerbations that contribute to morbidity and mortality in these disorders ${ }^{79,83,84}$.

\section{PAMP and DAMP signaling}

In addition to the multitiered innate defense system provided by mucociliary clearance and surfactant, epithelial cells recognize microbial pathogens and their products and initiate signaling to recruit and 'instruct' cells of the immune system (Fig. 3). The surfaces of respiratory epithelial cells provide the initial interface with the environment and are well equipped to respond to PAMPs and danger-associated molecular patterns (DAMPs). Pattern-recognition receptors (PRRs), including TLRs and NLRs, are widely expressed by respiratory epithelial cells ${ }^{85}$. While signaling by such receptors in hematopoietic cells is an essential component of innate immunity in the lungs, TLR signaling in structural, 
radioresistant cells, including airway epithelial cells, is critical for driving mucosal immune responses ${ }^{85-88}$. Conducting airway cells and type II alveolar cells express multiple TLRs, including TLR2 and TLR4; the latter is activated in response to lipopolysaccharide, respiratory syncytial virus, cigarette smoke and inflammatory cytokines ${ }^{89-91}$. Infection with the bacterial pathogen Klebsiella pneumoniae enhances the activity of TLR2 and TLR4 in human airway epithelial cells ${ }^{92}$. The activation of signaling pathways dependent on the nucleic-acid sensors RIG-I and TLR3 in the respiratory epithelium mediates host-defense responses to respiratory viral pathogens ${ }^{93-97}$. The NLRs Nod1 and Nod2, which are involved in the clearance of bacterial pathogens, are expressed in airway epithelial cells $^{98}$; however, less is known about epithelial cell-specific NLR signaling in the airways. TLR4-dependent activation of NF- $\kappa B$ by house dust mites and activation of PAR-2, a member of the proteinase-activated receptor (PAR) family, on respiratory epithelial cells by allergens cause epithelial secretion of chemokines and cytokines, such as IL-33, TSLP and IL-25, that influences the activation and recruitment of pulmonary dendritic cells, type 2 innate lymphoid cells (ILC2 cells or nuocytes) and $\mathrm{T}_{\mathrm{H}} 2$ lymphocytes ${ }^{88,99-106}$. An emerging body of literature supports the proposal of cooperation among TLRs, NLRs and PARs in mediating the innate responses of mucosal epithelial cells ${ }^{107,108}$.

Respiratory epithelial cells are well equipped with receptors that direct epithelial responses to molecules released during cell stress or necrosis. The DAMP PRR RAGE is expressed in respiratory epithelial cells, and type I alveolar cells show enrichment for this receptor ${ }^{109}$. The DAMP HMGB1 ('high-mobility group box 1'), a DNA-binding nuclear protein, is released during cell death and stimulation with cytokines. HMGB1 signals to nearby cells through TLR4-, TLR2- and RAGE-dependent pathways ${ }^{110}$. RAGE and TLRs act together in the regulation of cellular responses to inflammation, infection and cell stress ${ }^{110,111}$. Respiratory epithelial cells both respond to and produce endogenous DAMPs such as ATP, HMGB1, S100 proteins and uric acid ${ }^{112,113}$. Chronic exposure to pathogens, oxidants and toxicants causes the release of DAMPs that activate epithelial cell-intrinsic pattern-recognition pathways and also recruit and activate cells of the immune system that are active in chronic pulmonary disease, including cystic fibrosis and COPD ${ }^{114}$.

PAMPs and DAMPs regulate signaling cascades that alter epithelial gene expression and cytokine production. Signal transduction through the signaling adaptor MyD88 and MyD88-independent mechanisms activates NF-אB, MAP kinases and/or IRF3 in epithelial cells. The outcome of signaling via PAMPs or DAMPs is influenced by the nature of both the microbial pathogen and the inflammatory environment in the lungs and results in both protective effects and pathological effects on airway barrier functions. Epithelial cell-derived expression of cytokines and chemokines following the recognition of PAMPs or DAMPs influences the recruitment and activation of professional cells of the immune system to modulate inflammatory responses in the lungs ${ }^{85,115}$.

PRRs direct epithelial cell-intrinsic defense responses. Activation of signaling pathways of TLR2 and TLR3 by H. influenzae lipoprotein or rhinovirus activate the expression of MUC5AC in conducting airways ${ }^{116,117}$. Antimicrobial peptides produced by airway epithelial cells, such as the human $\beta$-defensins HBD- 2 and HDB- 4 , are expressed in response to activation of TLRs. Epithelial expression of HBD-2 is induced by microbes and is decreased in airways of people with $\mathrm{COPD}^{118}$. HBD-2 expression is activated via a TLR2- and NF- $\mathrm{KB}-$ dependent pathway ${ }^{119,120}$ and is linked to signaling by other TLRs, including TLR3, TLR4, TLR5 and TLR9 (refs. 120,121). TLR2 and TLR4 regulate both the secretion of diverse proteins of the innate immune system and the survival of respiratory epithelial cells following acute lung injury ${ }^{122}$. Collectively, the respiratory epithelium serves a critical role in the recognition of PAMPs and DAMPs in the airways and the subsequent initiation of pathways that regulate the responses of cells of the immune system, barrier function and the clearance of apoptotic cells and pathogens.

\section{Summary}

Innate defense responses of the respiratory epithelium have enabled evolutionary adaption to the constant exposure to microbial pathogens, particles and toxicants while maintaining lung function and tissue homeostasis. Respiratory epithelial cells produce a repertoire of biophysical scaffolds, host-defense molecules and barriers and communicate among themselves and with professional cells of the immune system through the production of cytokines, chemokines and DAMPs to maintain near sterility of the peripheral lungs throughout life.

\section{ACKNOWLEDGMENTS}

We thank B. Trapnell, J. Kitzmiller, T. Thatava and S. Wert for images and artwork, and A. Maher for manuscript preparation. Supported by the US National Institutes of Health (HL110964, HL122642 and HL110967 to J.A.W., and DK093784) and the Burroughs Wellcome Fund (T.A.).

\section{COMPETING FINANCIAL INTERESTS}

The authors declare no competing financial interests.

Reprints and permissions information is available online at http://www.nature.com/ reprints/index.html.

1. Metzger, R.J., Klein, O.D., Martin, G.R. \& Krasnow, M.A. The branching programme of mouse lung development. Nature 453, 745-750 (2008).

2. Kotton, D.N. \& Morrisey, E.E. Lung regeneration: mechanisms, applications and emerging stem cell populations. Nat. Med. 20, 822-832 (2014).

3. Hogan, B.L. et al. Repair and regeneration of the respiratory System: complexity, plasticity, and mechanisms of lung stem cell function. Cell Stem Cel/ 15, 123-138 (2014).

4. Tecle, T., Tripathi, S. \& Hartshorn, K.L. Review: Defensins and cathelicidins in lung immunity. Innate Immun. 16, 151-159 (2010)

5. Whitsett, J.A., Wert, S.E. \& Weaver, T.E. Alveolar surfactant homeostasis and the pathogenesis of pulmonary disease. Annu. Rev. Med. 61, 105-119 (2010).

6. Barkauskas, C.E. et al. Type 2 alveolar cells are stem cells in adult lung. J. Clin. Invest. 123, 3025-3036 (2013).

7. LaFemina, M.J. et al. Claudin-18 deficiency results in alveolar barrier dysfunction and impaired alveologenesis in mice. Am. J. Respir. Cell Mol. Biol. 51, 550-558 (2014).

8. Frank, J.A. Claudins and alveolar epithelial barrier function in the lung. Ann. NY Acad. Sci. 1257, 175-183 (2012).

9. Bhattacharya, J. \& Matthay, M.A. Regulation and repair of the alveolar-capillary barrier in acute lung injury. Annu. Rev. Physiol. 75, 593-615 (2013).

10. Koval, M. Claudin heterogeneity and control of lung tight junctions. Annu. Rev Physiol. 75, 551-567 (2013).

11. Koval, M. Differential pathways of claudin oligomerization and integration into tight junctions. Tissue Barriers 1, e24518 (2013).

12. Georas, S.N. \& Rezaee, F. Epithelial barrier function: At the front line of asthma immunology and allergic airway inflammation. J. Allergy Clin. Immunol. 134, 509520 (2014).

13. Grainge, C.L. \& Davies, D.E. Epithelial injury and repair in airways diseases. Chest 144, 1906-1912 (2013)

14. Kreda, S.M., Davis, C.W. \& Rose, M.C. CFTR, mucins, and mucus obstruction in cystic fibrosis. Cold Spring Harb. Perspect. Med. 2, a009589 (2012).

15. Voynow, J.A. \& Rubin, B.K. Mucins, mucus, and sputum. Chest 135, 505-512 (2009).

16. Button, B. et al. A periciliary brush promotes the lung health by separating the mucus layer from airway epithelia. Science 337, 937-941 (2012).

17. Williams, O.W., Sharafkhaneh, A., Kim, V., Dickey, B.F. \& Evans, C.M. Airway mucus: from production to secretion. Am. J. Respir. Cell Mol. Biol. 34, 527-536 (2006).

18. Thai, P., Loukoianov, A., Wachi, S. \& Wu, R. Regulation of airway mucin gene expression. Annu. Rev. Physiol. 70, 405-429 (2008).

19. Fujisawa, $\mathrm{T}$. et al. NF-KB mediates IL-1 $\beta$ - and IL-17A-induced MUC5B expression in airway epithelial cells. Am. J. Respir. Cell Mol. Biol. 45, 246-252 (2011).

20. Erle, D.J. \& Sheppard, D. The cell biology of asthma. J. Cell Biol. 205, 621-631 (2014).

21. Hasnain, S.Z. et al. Muc5ac: a critical component mediating the rejection of enteric nematodes. J. Exp. Med. 208, 893-900 (2011).

22. Koeppen, $\mathrm{M}$. et al. Detrimental role of the airway mucin Muc5ac during ventilatorinduced lung injury. Mucosal Immunol. 6, 762-775 (2013).

23. Ehre, C. et al. Overexpressing mouse model demonstrates the protective role of Muc5ac in the lungs. Proc. Natl. Acad. Sci. USA 109, 16528-16533 (2012).

24. Roy, M.G. et al. Muc5b is required for airway defence. Nature 505, 412-416 (2014). 
25. Hoegger, M.J. et al. Assessing mucociliary transport of single particles in vivo shows variable speed and preference for the ventral trachea in newborn pigs. Proc. Natl. Acad. Sci. USA 111, 2355-2360 (2014).

26. Henderson, A.G. et al. Cystic fibrosis airway secretions exhibit mucin hyperconcentration and increased osmotic pressure. J. Clin. Invest. 124, 3047-3060 (2014).

27. Matsui, $\mathrm{H}$. et al. A physical linkage between cystic fibrosis airway surface dehydration and Pseudomonas aeruginosa biofilms. Proc. Natl. Acad. Sci. USA 103. 18131-18136 (2006).

28. Luan, X. et al. Pseudomonas aeruginosa triggers CFTR-mediated airway surface liquid secretion in swine trachea. Proc. Natl. Acad. Sci. USA 111, 12930-12935 (2014).

29. Guseh, J.S. et al. Notch signaling promotes airway mucous metaplasia and inhibits alveolar development. Development 136, 1751-1759 (2009).

30. Rock, J.R. et al. Notch-dependent differentiation of adult airway basal stem cells. Cell Stem Cell 8, 639-648 (2011).

31. Morimoto, M., Nishinakamura, R., Saga, Y. \& Kopan, R. Different assemblies of Notch receptors coordinate the distribution of the major bronchial Clara, ciliated and neuroendocrine cells. Development 139, 4365-4373 (2012).

32. Chen, G. et al. SPDEF is required for mouse pulmonary goblet cell differentiation and regulates a network of genes associated with mucus production. J. Clin. Invest. 119, 2914-2924 (2009).

33. Scudieri, P. et al. Association of TMEM16A chloride channel overexpression with airway goblet cell metaplasia. J. Physiol. (Lond.) 590, 6141-6155 (2012).

34. Huang, F. et al. Calcium-activated chloride channel TMEM16A modulates mucin secretion and airway smooth muscle contraction. Proc. Natl. Acad. Sci. USA 109, 16354-16359 (2012).

35. Maeda, Y. et al. Airway epithelial transcription factor NK2 homeobox 1 inhibits mucous cell metaplasia and Th2 inflammation. Am. J. Respir. Crit. Care Med. 184 421-429 (2011)

36. Alevy, Y.G. et al. IL-13-induced airway mucus production is attenuated by MAPK13 inhibition. J. Clin. Invest. 122, 4555-4568 (2012).

37. Chen, G. et al. Foxa3 induces goblet cell metaplasia and inhibits innate antiviral immunity. Am. J. Respir. Crit. Care Med. 189, 301-313 (2014).

38. Korfhagen, T.R. et al. SAM-pointed domain ETS factor mediates epithelial cellintrinsic innate immune signaling during airway mucous metaplasia. Proc. Natl. Acad. Sci. USA 109, 16630-16635 (2012).

39. Popatia, R., Haver, K. \& Casey, A. Primary ciliary dyskinesia: an update on new diagnostic modalities and review of the literature. Pediatr. Allergy Immunol. Pulmonol. 27, 51-59 (2014).

40. Knowles, M.R., Daniels, L.A., Davis, S.D., Zariwala, M.A. \& Leigh, M.W. Primary ciliary dyskinesia. Recent advances in diagnostics, genetics, and characterization of clinical disease. Am. J. Respir. Crit. Care Med. 188, 913-922 (2013).

41. McKenzie, C.W. et al. Enhanced response to pulmonary Streptococcus pneumoniae infection is associated with primary ciliary dyskinesia in mice lacking Pcdp1 and Spef2. Cilia 2, 18 (2013).

42. Choksi, S.P., Lauter, G., Swoboda, P. \& Roy, S. Switching on cilia: transcriptional networks regulating ciliogenesis. Development 141, 1427-1441 (2014).

43. Cloonan, S.M., Lam, H.C., Ryter, S.W. \& Choi, A.M. "Ciliophagy": the consumption of cilia components by autophagy. Autophagy 10, 532-534 (2014).

44. Bou Saab, J., Losa, D., Chanson, M. \& Ruez, R. Connexins in respiratory and gastrointestinal mucosal immunity. FEBS Lett. 588, 1288-1296 (2014).

45. Martin, F.J. \& Prince, A.S. TLR2 regulates gap junction intercellular communication in airway cells. J. Immunol. 180, 4986-4993 (2008).

46. Hartshorn, K.L. Role of surfactant protein A and D (SP-A and SP-D) in human antiviral host defense. Front. Biosci. 2, 527-546 (2010).

47. Ariki, S., Nishitani, C. \& Kuroki, Y. Diverse functions of pulmonary collectins in host defense of the lung. J.Biomed. Biotechnol. 2012, 532071 (2012).

48. Numata, M., Chu, H.W., Dakhama, A. \& Voelker, D.R. Pulmonary surfactant phosphatidylglycerol inhibits respiratory syncytial virus-induced inflammation and infection. Proc. Natl. Acad. Sci. USA 107, 320-325 (2010).

49. Numata, M. et al. Phosphatidylglycerol provides short-term prophylaxis against respiratory syncytial virus infection. J. Lipid Res. 54, 2133-2143 (2013).

50. Yang, L. et al. Surfactant protein B propeptide contains a saposin-like protein domain with antimicrobial activity at low pH. J. Immunol. 184, 975-983 (2010).

51. Ryan, M.A. et al. Antimicrobial activity of native and synthetic surfactant protein $B$ peptides. J. Immunol. 176, 416-425 (2006).

52. Augusto, L.A., Li, J., Synguelakis, M., Johansson, J. \& Chaby, R. Structural basis for interactions between lung surfactant protein $\mathrm{C}$ and bacterial lipopolysaccharide. J. Biol. Chem. 277, 23484-23492 (2002).

53. Glasser, S.W. et al. Persistence of LPS-induced lung inflammation in surfactant protein-C-deficient mice. Am. J. Respir. Cell Mol. Biol. 49, 845-854 (2013).

54. Glasser, S.W. et al. Surfactant protein C-deficient mice are susceptible to respiratory syncytial virus infection. Am. J. Physiol. Lung Cell. Mol. Physiol. 297, L64-L72 (2009)

55. McCormack, F.X. \& Whitsett, J.A. The pulmonary collectins, SP-A and SP-D, orchestrate innate immunity in the lung. J. Clin. Invest. 109, 707-712 (2002).

56. Trapnell, B.C., Whitsett, J.A. \& Nakata, K. Pulmonary alveolar proteinosis. N. Engl. J. Med. 349, 2527-2539 (2003)

57. Guilliams, M. et al. Alveolar macrophages develop from fetal monocytes that differentiate into long-lived cells in the first week of life via GM-CSF. J. Exp. Med. 210 1977-1992 (2013)

58. Berclaz, P.Y. et al. GM-CSF regulates a PU.1-dependent transcriptional program determining the pulmonary response to LPS. Am. J. Respir. Cell Mol. Biol. 36, 114-121 (2007)
59. Suzuki, T. et al. Hereditary pulmonary alveolar proteinosis: pathogenesis, presentation, diagnosis, and therapy. Am. J. Respir. Crit. Care Med. 182, 1292-1304 (2010).

60. Martinez-Moczygemba, M. et al. Pulmonary alveolar proteinosis caused by deletion of the GM-CSFRalpha gene in the X chromosome pseudoautosomal region 1. J. Exp. Med. 205, 2711-2716 (2008).

61. Suzuki, T. et al. Familial pulmonary alveolar proteinosis caused by mutations in CSF2RA. J. Exp. Med. 205, 2703-2710 (2008).

62. Suzuki, T. et al. Hereditary pulmonary alveolar proteinosis caused by recessive CSF2RB mutations. Eur. Respir. J. 37, 201-204 (2011).

63. Suzuki, T. et al. Pulmonary macrophage transplantation therapy. Nature 514, 450 454 (2014)

64. Trapnell, B.C., Carey, B.C., Uchida, K. \& Suzuki, T. Pulmonary alveolar proteinosis, a primary immunodeficiency of impaired GM-CSF stimulation of macrophages. Curr. Opin. Immunol. 21, 514-521 (2009).

65. Westphalen, K. et al. Sessile alveolar macrophages communicate with alveolar epithelium to modulate immunity. Nature 506, 503-506 (2014).

66. Osorio, F., Lambrecht, B. \& Janssens, S. The UPR and lung disease. Semin Immunopathol. 35, 293-306 (2013).

67. Choi, A.M., Ryter, S.W. \& Levine, B. Autophagy in human health and disease. N. Engl. J. Med. 368, 651-662 (2013).

68. Nakahira, K. \& Choi, A.M. Autophagy: a potential therapeutic target in lung diseases. Am. J. Physiol. Lung Cell. Mol. Physiol. 305, L93-L107 (2013).

69. Heaton, N.S. et al. Long-term survival of influenza virus infected club cells drives immunopathology. J. Exp. Med. 211, 1707-1714 (2014).

70. Juncadella, I.J. et al. Apoptotic cell clearance by bronchial epithelial cells critically influences airway inflammation. Nature 493, 547-551 (2013)

71. Maguire, J.A., Mulugeta, S. \& Beers, M.F. Endoplasmic reticulum stress induced by surfactant protein $\mathrm{C}$ BRICHOS mutants promotes proinflammatory signaling by epithelial cells. Am. J. Respir. Cell Mol. Biol. 44, 404-414 (2011).

72. Steele, M.P. \& Schwartz, D.A. Molecular mechanisms in progressive idiopathic pul monary fibrosis. Annu. Rev. Med. 64, 265-276 (2013).

73. Korfei, M. et al. Epithelial endoplasmic reticulum stress and apoptosis in sporadic idiopathic pulmonary fibrosis. Am. J. Respir. Crit. Care Med. 178, 838-846 (2008).

74. Tanjore, H., Blackwell, T.S. \& Lawson, W.E. Emerging evidence for endoplasmic reticulum stress in the pathogenesis of idiopathic pulmonary fibrosis. Am. J. Physiol. Lung Cell. Mol. Physiol. 302, L721-L729 (2012)

75. Fingerlin, T.E. et al. Genome-wide association study identifies multiple susceptibility loci for pulmonary fibrosis. Nat. Genet. 45, 613-620 (2013).

76. Martino, M.B. et al. The ER stress transducer IRE1 $\beta$ is required for airway epithelia mucin production. Mucosal Immunol. 6, 639-654 (2013).

77. Akei, H. et al. Surface tension influences cell shape and phagocytosis in alveolar macrophages. Am. J. Physiol. Lung Cell. Mol. Physiol. 291, L572-L579 (2006).

78. Glasser, S.W. et al. Pneumonitis and emphysema in $\mathrm{sp}-\mathrm{C}$ gene targeted mice. J. Biol. Chem. 278, 14291-14298 (2003).

79. Morris, A. et al. Comparison of the respiratory microbiome in healthy nonsmokers and smokers. Am. J. Respir. Crit. Care Med. 187, 1067-1075 (2013).

80. Dickson, R.P., Erb-Downward, J.R. \& Huffnagle, G.B. The role of the bacterial microbiome in lung disease. Expert Rev. Respir. Med. 7, 245-257 (2013).

81. Busse, W.W., Lemanske, R.F. Jr. \& Gern, J.E. Role of viral respiratory infections in asthma and asthma exacerbations. Lancet 376, 826-834 (2010).

82. Ege, M.J. et al. Exposure to environmental microorganisms and childhood asthma. N. Engl. J. Med. 364, 701-709 (2011).

83. Sze, M.A. et al. The lung tissue microbiome in chronic obstructive pulmonary disease. Am. J. Respir. Crit. Care Med. 185, 1073-1080 (2012).

84. Hansel, T.T., Johnston, S.L. \& Openshaw, P.J. Microbes and mucosal immune responses in asthma. Lancet $\mathbf{3 8 1 , 8 6 1 - 8 7 3 ~ ( 2 0 1 3 ) . ~}$

85. Lambrecht, B.N. \& Hammad, H. The airway epithelium in asthma. Nat. Med. 18 684-692 (2012).

86. Cleaver, J.O. et al. Lung epithelial cells are essential effectors of inducible resistance to pneumonia. Mucosal Immunol. 7, 78-88 (2014).

87. Van Maele, L. et al. Airway structural cells regulate TLR5-mediated mucosal adjuvant activity. Mucosal Immunol. 7, 489-500 (2014)

88. Hammad, H. et al. House dust mite allergen induces asthma via Toll-like receptor 4 triggering of airway structural cells. Nat. Med. 15, 410-416 (2009).

89. Armstrong, L. et al. Expression of functional toll-like receptor-2 and -4 on alveola epithelial cells. Am. J. Respir. Cell Mol. Biol. 31, 241-245 (2004).

90. Monick, M.M. et al. Respiratory syncytial virus up-regulates TLR4 and sensitizes airway epithelial cells to endotoxin. J. Biol. Chem. 278, 53035-53044 (2003).

91. Pace, E. et al. Cigarette smoke increases Toll-like receptor 4 and modifies lipopoly saccharide-mediated responses in airway epithelial cells. Immunology 124, 401-411 (2008).

92. Regueiro, V. et al. Klebsiella pneumoniae increases the levels of Toll-like receptors 2 and 4 in human airway epithelial cells. Infect. Immun. 77, 714-724 (2009).

93. Wang, Q. et al. Role of double-stranded RNA pattern recognition receptors in rhinovirusinduced airway epithelial cell responses. J. Immunol. 183, 6989-6997 (2009).

94. Bertolusso, R. et al. Dynamic cross talk model of the epithelial innate immune response to double-stranded RNA stimulation: coordinated dynamics emerging from cell-level noise. PLOS ONE 9, e93396 (2014).

95. Groskreutz, D.J. et al. Respiratory syncytial virus induces TLR3 protein and protein kinase $R$, leading to increased double-stranded RNA responsiveness in airway epithelial cells. J. Immunol. 176, 1733-1740 (2006). 
96. Le Goffic, $R$, et al. Cutting edge: influenza A virus activates TLR3-dependent inflammatory and RIG-I-dependent antiviral responses in human lung epithelial cells. J. Immunol. 178, 3368-3372 (2007).

97. Shornick, L.P. et al. Airway epithelial versus immune cell Stat1 function for innate defense against respiratory viral infection. J. Immunol. 180, 3319-3328 (2008).

98. Uehara, A., Fujimoto, Y., Fukase, K. \& Takada, H. Various human epithelial cells express functional Toll-like receptors, NOD1 and NOD2 to produce anti-microbial peptides, but not proinflammatory cytokines. Mol. Immunol. 44, 3100-3111 (2007).

99. Tan, A.M. et al. TLR4 signaling in stromal cells is critical for the initiation of allergic Th2 responses to inhaled antigen. J. Immunol. 184, 3535-3544 (2010).

100. Li DQ, Zhang L, Pflugfelder SC, De Paiva CS, Zhang X, Zhao G, et al. Short ragweed pollen triggers allergic inflammation through Toll-like receptor 4-dependent thymic stromal lymphopoietin/OX40 ligand/OX40 signaling pathways. J. Allergy Clin. Immunol. 128, 1318-1325 (2011).

101. Willart, M.A. et al. Interleukin-1 $\alpha$ controls allergic sensitization to inhaled house dust mite via the epithelial release of GM-CSF and IL-33. J. Exp. Med. 209, 1505-1517 (2012)

102. Kouzaki, H., O'Grady, S.M., Lawrence, C.B. \& Kita, H. Proteases induce production of thymic stromal lymphopoietin by airway epithelial cells through protease-activated receptor-2. J. Immunol. 183, 1427-1434 (2009).

103. Kouzaki, H., Tojima, I., Kita, H. \& Shimizu, T. Transcription of interleukin-25 and extracellular release of the protein is regulated by allergen proteases in airway epithelial cells. Am. J. Respir. Cell Mol. Biol. 49, 741-750 (2013).

104. Lambrecht, B.N. \& Hammad, H. Allergens and the airway epithelium response: Gateway to allergic sensitization. J. Allergy Clin. Immunol. 134, 499-507 (2014).

105. Hill, D.A. et al. Commensal bacteria-derived signals regulate basophil hematopoiesis and allergic inflammation. Nat. Med. 18, 538-546 (2012).

106. Monticelli, L.A., Sonnenberg, G.F. \& Artis, D. Innate lymphoid cells: critical regulators of allergic inflammation and tissue repair in the lung. Curr. Opin. Immunol. 24, 284-289 (2012).

107. Nhu, Q.M. et al. Novel signaling interactions between proteinase-activated receptor 2 and Toll-like receptors in vitro and in vivo. Mucosal Immunol. 3, 29-39 (2010).

108. Gieseler, F., Ungefroren, H., Settmacher, U., Hollenberg, M.D. \& Kaufmann, R. Proteinase-activated receptors (PARs) - focus on receptor-receptor-interactions and their physiological and pathophysiological impact. Cell Commun. Signal. 11, 86 (2013).

109. Dahlin, K et al. Identification of genes differentially expressed in rat alveolar type I cells. Am. J. Respir. Cell Mol. Biol. 31, 309-316 (2004).

110. Sims, G.P., Rowe, D.C., Rietdijk, S.T., Herbst, R. \& Coyle, A.J. HMGB1 and RAGE in inflammation and cancer. Annu. Rev. Immunol. 28, 367-388 (2010).

111. Ibrahim, Z.A., Armour, C.L., Phipps, S. \& Sukkar, M.B. RAGE and TLRs: relatives, friends or neighbours? Mol. Immunol. 56, 739-744 (2013).

112. Kang, J.H., Hwang, S.M. \& Chung, I.Y. S100A8, S100A9, and S100A12 activate airway epithelial cells to produce MUC5AC via ERK and NF-אB pathways. Immunology doi:10.1111/imm.12352 (30 June 2014).

113. Ellson, C.D., Dunmore, R., Hogaboam, C.M., Sleeman, M.A. \& Murray, L.A. Dangerassociated molecular patterns and danger signals in idiopathic pulmonary fibrosis. Am. J. Respir. Cell Mol. Biol. 51, 163-168 (2014).

114. Pouwels, S.D. et al. DAMPs activating innate and adaptive immune responses in COPD. Mucosal Immunol. 7, 215-226 (2014).

115. Parker, D. \& Prince, A. Type I interferon response to extracellular bacteria in the airway epithelium. Trends Immunol. 32, 582-588 (2011).

116. Chen, R. et al. Nontypeable Haemophilus influenzae lipoprotein P6 induces MUC5AC mucin transcription via TLR2-TAK1-dependent p38 MAPK-AP1 and IKK $\beta$ - IKB NF-kB signaling pathways. Biochem. Biophys. Res. Commun. 324, 1087-1094 (2004).

117. Zhu, L et al. Rhinovirus-induced major airway mucin production involves a novel TLR3-EGFR-dependent pathway. Am. J. Respir. Cell Mol. Biol. 40, 610-619 (2009).

118. Pace, E. et al. Beta defensin-2 is reduced in central but not in distal airways of smoker COPD patients. PLoS ONE 7, e33601 (2012).

119. Wang, $X$. et al. Airway epithelia regulate expression of human $\beta$-defensin 2 through Toll-like receptor 2. FASEB J. 17, 1727-1729 (2003)

120. Hertz, C.J. et al. Activation of Toll-like receptor 2 on human tracheobronchial epithelial cells induces the antimicrobial peptide human b defensin-2. J. Immunol. 171, 6820-6826 (2003).

121. Parker, D. \& Prince, A. Innate immunity in the respiratory epithelium. Am. J. Respir. Cell Mol. Biol. 45, 189-201 (2011).

122. Jiang, D. et al. Regulation of lung injury and repair by Toll-like receptors and hyaluronan. Nat. Med. 11, 1173-1179 (2005). 